\title{
The Complexity of Approximating the Matching Polynomial in the Complex Plane
}

\author{
Ivona Bezáková
}

Department of Computer Science, Rochester Institute of Technology, Rochester, NY, USA

ib@cs.rit.edu

\author{
Andreas Galanis \\ Department of Computer Science, University of Oxford, UK \\ andreas.galanis@cs.ox.ac.uk
}

Leslie Ann Goldberg

Department of Computer Science, University of Oxford, UK

leslie.goldberg@cs.ox.ac.uk

\section{Daniel Štefankovič}

Department of Computer Science, University of Rochester, Rochester, NY, USA

stefanko@cs.rochester.edu

\begin{abstract}
We study the problem of approximating the value of the matching polynomial on graphs with edge parameter $\gamma$, where $\gamma$ takes arbitrary values in the complex plane.

When $\gamma$ is a positive real, Jerrum and Sinclair showed that the problem admits an FPRAS on general graphs. For general complex values of $\gamma$, Patel and Regts, building on methods developed by Barvinok, showed that the problem admits an FPTAS on graphs of maximum degree $\Delta$ as long as $\gamma$ is not a negative real number less than or equal to $-1 /(4(\Delta-1))$. Our first main result completes the picture for the approximability of the matching polynomial on bounded degree graphs. We show that for all $\Delta \geq 3$ and all real $\gamma$ less than $-1 /(4(\Delta-1))$, the problem of approximating the value of the matching polynomial on graphs of maximum degree $\Delta$ with edge parameter $\gamma$ is \#P-hard.

We then explore whether the maximum degree parameter can be replaced by the connective constant. Sinclair et al. showed that for positive real $\gamma$ it is possible to approximate the value of the matching polynomial using a correlation decay algorithm on graphs with bounded connective constant (and potentially unbounded maximum degree). We first show that this result does not extend in general in the complex plane; in particular, the problem is \#P-hard on graphs with bounded connective constant for a dense set of $\gamma$ values on the negative real axis. Nevertheless, we show that the result does extend for any complex value $\gamma$ that does not lie on the negative real axis. Our analysis accounts for complex values of $\gamma$ using geodesic distances in the complex plane in the metric defined by an appropriate density function.
\end{abstract}

2012 ACM Subject Classification Theory of computation $\rightarrow$ Approximation algorithms analysis; Theory of computation $\rightarrow$ Problems, reductions and completeness

Keywords and phrases matchings, partition function, correlation decay, connective constant

Digital Object Identifier 10.4230/LIPIcs.ICALP.2019.22

Category Track A: Algorithms, Complexity and Games

Related Version A full version of the paper is available at http://arxiv.org/abs/1807.04930. The theorem numbering here matches the full version.

Funding Ivona Bezáková: Research supported by NSF grant CCF-1319987.

Andreas Galanis: The research leading to these results has received funding from the European Research Council under the European Union's Seventh Framework Programme (FP7/2007-2013) ERC grant agreement no. 334828. The paper reflects only the authors' views and not the views of the ERC or the European Commission. The European Union is not liable for any use that may be made of the information contained therein.

cc (i) () Ivona Bezáková, Andreas Galanis, Leslie A. Goldberg, and Daniel Štefankovič; c. licensed under Creative Commons License CC-BY

46th International Colloquium on Automata, Languages, and Programming (ICALP 2019). Editors: Christel Baier, Ioannis Chatzigiannakis, Paola Flocchini, and Stefano Leonardi; Article No. 22; pp. 22:1-22:13

Leibniz International Proceedings in Informatics

LIPICS Schloss Dagstuhl - Leibniz-Zentrum für Informatik, Dagstuhl Publishing, Germany 
Leslie Ann Goldberg: The research leading to these results has received funding from the European Research Council under the European Union's Seventh Framework Programme (FP7/2007-2013) ERC grant agreement no. 334828. The paper reflects only the authors' views and not the views of the ERC or the European Commission. The European Union is not liable for any use that may be made of the information contained therein.

Daniel Štefankovič: Research supported by NSF grant CCF-1563757.

\section{Introduction}

We study the problem of approximating the matching polynomial of a graph. This polynomial has a parameter $\gamma$, called the edge activity. A matching of a graph $G$ is a set $M \subseteq E(G)$ such that each vertex $v \in V(G)$ is contained in at most one edge in $M$. We denote by $\mathcal{M}_{G}$ the set of all matchings of $G$. The matching polynomial $Z_{G}(\gamma)$ is given by

$$
Z_{G}(\gamma)=\sum_{M \in \mathcal{M}_{G}} \gamma^{|M|}
$$

This polynomial is also referred to as the partition function of the monomer-dimer model in statistical physics.

Here is what is known about approximating this polynomial. We first describe the case where $\gamma$ is positive and real. This is a natural case, and is the case where the first complexity-theoretic results were obtained. We next describe the more general case, where $\gamma$ is a complex number. There are many reasons for considering the more general case. The parameter $\gamma$ is defined to be complex, rather than real, in the classic paper of Heilmann and Lieb [8]. Furthermore, it has recently been shown [15] that the quantum evolution of a system originally in thermodynamic equilibrium is equivalent to the partition function of the system with a complex parameter. As [15] explains, recent discoveries in physics make it possible to study thermodynamics in the complex plane of physical parameters - so complex parameters are increasingly relevant. As we will see in this paper, it is beneficial to study partition functions with complex parameters even when one is most interested in the real case - the reason is that the generalisation sheds light on "what is really going on" with complexity bottlenecks, and on appropriate potential functions. Here is the summary of known results in both cases.

- When the edge activity $\gamma$ is a positive real number: For any positive real number $\gamma$, Jerrum and Sinclair [9, Corollary 4.4] gave an FPRAS for approximating $Z_{G}(\gamma)$. Using the correlation decay technique, Bayati et al. [3] gave a (deterministic) FPTAS for the same problem for the case in which the degree of the input graph $G$ is at most a constant $\Delta$.

- When the edge activity $\gamma$ is a complex number: Known results are restricted to the case where $\gamma$ is not a real number less than or equal to $-1 /(4(\Delta-1))$. In this case, there is a positive result, due to Patel and Regts [11]. Using a method of Barvinok [1,2] for approximating a partition function by truncating its Taylor series (in a region where the partition function has no zeroes), Patel and Regts [11, Theorem 1.2] extended the positive result of Bayati et al. to the case in which $\gamma$ is a complex number that is not a negative real that is less than $-1 /(4(\Delta-1))$, see also [2, Section 5.1.7]). Patel and Regts obtained a polynomial time algorithm (rather than a quasi-polynomial time one) by developing clever methods for exactly computing coefficients of the Taylor series. 
Our first contribution completes this picture by showing that for all $\Delta \geq 3$ and all real $\gamma<-1 /(4(\Delta-1))$ it is actually \#P-hard to approximate $Z_{G}(\gamma)$ on graphs with degree at most $\Delta$. We use the following notation to state our result more precisely. We consider the problems of multiplicatively approximating the norm of $Z_{G}(\gamma)$, and of computing its sign. Our first theorem shows that, for all $\Delta \geq 3$ and all rational numbers $\gamma<-1 /(4(\Delta-1))$, it is \#P-hard to approximate $\left|Z_{G}(\gamma)\right|$ on bipartite graphs of maximum degree $\Delta$ within a constant factor.

- Theorem 1. Let $\Delta \geq 3$ and $\gamma<-\frac{1}{4(\Delta-1)}$ be a rational number. Then, it is \#P-hard to approximate $\left|Z_{G}(\gamma)\right|$ within a factor of 1.01 on graphs $G$ of maximum degree $\Delta$, even when restricted to bipartite graphs $G$ with $Z_{G}(\gamma) \neq 0$.

The number 1.01 in Theorem 1 is not important. It can be replaced with any constant greater than 1. In fact, for any fixed $\epsilon>0$, the theorem, together with a standard powering argument, shows that it is \#P-hard to approximate $Z_{G}(\gamma)$ within a factor of $2^{|V(G)|^{1-\epsilon}}$.

Our second theorem shows that it is \#P-hard to compute the sign of $Z_{G}(\gamma)$ on bipartite graphs of maximum degree $\Delta$.

Theorem 2. Let $\Delta \geq 3$ and $\gamma<-\frac{1}{4(\Delta-1)}$ be a rational number. Then, it is \#P-hard to decide whether $Z_{G}(\gamma)>0$ on graphs $G$ of maximum degree $\Delta$, even when restricted to bipartite graphs $G$ with $Z_{G}(\gamma) \neq 0$.

We next explore whether the bound on the maximum degree of $G$ can be relaxed to a restriction on average degree. The notion of average degree that we use is the connective constant. Given a graph $G$, and a vertex $v$, let $N_{G}(v, k)$ be the number of $k$-edge paths in $G$ that start from $v$. The following definition is taken almost verbatim from $[13,14] .^{1}$

- Definition $3([13,14])$. Let $\mathcal{F}$ be a family of finite graphs and let $\Delta$, a and $c$ be positive real numbers. The connective constant of $\mathcal{F}$ is at most $\Delta$ with profile $(a, c)$ if, for any graph $G=(V, E)$ in $\mathcal{F}$ and any vertex $v$ in $G$, it holds that $\sum_{k=1}^{\ell} N_{G}(v, k) \leq c \Delta^{\ell}$ for all $\ell \geq a \log |V|$.

Sinclair, Srivastava, Štefankovič and Yin [13, Theorem 1.3] showed that, for fixed $\Delta$, when $\gamma$ is a positive real, the correlation decay method gives an FPTAS for approximating $Z_{G}(\gamma)$ on graphs $G$ with connective constant at most $\Delta$ (without any bound on the maximum degree of $G)$. The run-time of their algorithm is $(n / \epsilon)^{\mathrm{O}(\sqrt{\gamma \Delta} \log \Delta)}$, where $n$ is the number of vertices of $G$ and $\epsilon$ is the relative error.

Our next result shows that, in striking contrast to the bounded-degree case, the algorithmic result of Sinclair et al. cannot be extended to negative reals, even if $\gamma \geq-1 /(4(\Delta-1))$. Given positive real numbers $a$ and $c$ and a real number $\Delta>1$, let $\mathcal{F}_{\Delta, a, c}$ be the set of graphs with connective constant at most $\Delta$ and profile $(a, c)$.

- Theorem 4. There exist a dense set of values $\gamma$ on the negative real axis such that the following holds for any real numbers $\Delta>1$ and all $a, c>0$.

1. It is \#P-hard to approximate $\left|Z_{G}(\gamma)\right|$ within a factor 1.01 on graphs $G \in \mathcal{F}_{\Delta, a, c}$,

2. it is \#P-hard to decide whether $Z_{G}(\gamma)>0$ on graphs $G \in \mathcal{F}_{\Delta, a, c}$.

Both of these results hold even when restricted to bipartite graphs $G$ with $Z_{G}(\gamma) \neq 0$.

1 The only difference between Definition 3 and the corresponding definitions in $[13,14]$ is the addition of the terminology "profile $(a, c)$ " which will be used to state our hardness results in a strong form (the results in $[13,14]$ were algorithmic which is why this handle on the constants $a$ and $c$ was not required). 
The algorithmic contribution of our paper is to show that, despite the hardness result of Theorem 4, correlation decay gives a good approximation algorithm for any complex value $\gamma$ that does not lie on the negative real axis when the input graph has bounded connective constant. It is interesting that we are able to use correlation decay to get a good approximation for all non-real complex values $\gamma$. Our result is the only known approximation in this setting. In particular, it is not known how to obtain such a result using the method of Patel and Regts [11]. In order to describe our result, we use the following notation. Given a complex number $x$, let $\arg (x)$ denote the principal value of its argument in the range $[0,2 \pi)$ and $|x|$ denote its norm. Our result is the following.

- Theorem 5. Let $\Delta$, a and $c$ be positive real numbers and let $\gamma \in \mathbb{C} \backslash \mathbb{R}_{<0}$ be any fixed edge activity. Then there is an algorithm which takes as input an n-vertex graph $G \in \mathcal{F}_{\Delta, a, c}$ and a rational $\epsilon \in(0,1)$ and produces an output $\hat{Z}=Z_{G}(\gamma) \mathrm{e}^{z}$ for some complex number $z$ with $|z| \leq \epsilon$. The running time of the algorithm is $(\hat{c} n / \epsilon)^{O((1+a+\sqrt{|\hat{\gamma}| \Delta}) \log \Delta)}$ where $\hat{\gamma}=\frac{2|\gamma|}{1+\cos (\arg \gamma)}$ and $\hat{c}=\max \{1, c\}$.

Theorem 5 gives an algorithmic result which contrasts with the hardness results of Theorems 1 and 2. It has the following corollary.

- Corollary 6. Let $\Delta$, $a$ and $c$ be positive real numbers and let $\gamma \in \mathbb{C} \backslash \mathbb{R}_{<0}$ be any fixed edge activity. Then, for any rational $K>1$ and any positive rational $\rho$, there are polynomialtime algorithms to take as input a graph $G \in \mathcal{F}_{\Delta, a, c}$ and approximate $\left|Z_{G}(\gamma)\right|$ within a multiplicative factor of $K$ and $\arg \left(Z_{G}(\gamma)\right)$ within an additive error $\rho$.

In order to prove Theorem 5, showing correlation decay for complex $\gamma$, we use geodesic distances in the complex plane in the metric defined by an appropriate density function. Correlation decay for complex activities has been analysed in the context of the hard-core model (see Harvey, Srivastava and Vondrák [7]) ${ }^{2}$. The region in the complex plane in which the authors of [7] worked allowed them to measure distances using the norm instead of requiring geodesic distances. An alternative approach was given by Peters and Regts [12], again in the context of the hard-core model, where they showed contraction within the basin of an attracting fixpoint using the theory of complex dynamical systems.

\section{Preliminaries}

Let $\gamma$ be a complex number and $G=(V, E)$ be an arbitrary graph. Recall that $\mathcal{M}_{G}$ is the set of matchings of $G$. For a matching $M \in \mathcal{M}_{G}$, we denote by $\operatorname{ver}(M)$ the set of matched vertices in the matching $M$. For a vertex $u$ in $G$, we also define

$$
Z_{G, u}(\gamma):=\sum_{M \in \mathcal{M}_{G} ; u \in \operatorname{ver}(M)} \gamma^{|M|} \text { and } Z_{G, \neg u}(\gamma):=\sum_{M \in \mathcal{M}_{G} ; u \notin \operatorname{ver}(M)} \gamma^{|M|} .
$$

Thus, $Z_{G, u}(\gamma)$ is the contribution to the partition function $Z_{G}(\gamma)$ from those matchings $M \in \mathcal{M}_{G}$ such that $u$ is matched in $M$, while $Z_{G, \neg u}(\gamma)$ is the contribution to the partition function $Z_{G}(\gamma)$ from those matchings $M \in \mathcal{M}_{G}$ such that $u$ is not matched in $M$.

We will use the following result about the location of the zeroes of the matching polynomial.

2 Note that Harvey et al were actually working with the mutivariate hard-core polynomial - this causes interesting complications which will not be relevant for this paper. They also extend their method (for the hard-core polynomial, in their region) to graphs of unbounded degree that have bounded connective constant. 
Theorem 7 ([8], see, e.g., [2, Theorem 5.1.2]). Let $\Delta \geq 3$ be an integer and $G$ be a graph of maximum degree $\Delta$. Then, for all complex $\gamma$ that do not lie on the interval $\left(-\infty,-\frac{1}{4(\Delta-1)}\right)$ of the negative real axis, it holds that $Z_{G}(\gamma) \neq 0$.

Corollary 8. Let $\Delta \geq 3$ be an integer and $\gamma>-\frac{1}{4(\Delta-1)}$ be a real number. Then, for all graphs $G$ of maximum degree $\Delta$ it holds that $Z_{G}(\gamma)>0$.

For our approximation algorithm of Theorem 5 , given a graph $G=(V, E)$ with $Z_{G}(\gamma) \neq 0$ and a vertex $v \in V$, we will be interested in the quantity

$$
p_{v}(G, \gamma):=Z_{G, \neg v}(\gamma) / Z_{G}(\gamma)
$$

The algorithm will be based on the following result by Godsil.

- Theorem 9 ([6]). Let $\gamma \in \mathbb{C} \backslash \mathbb{R}_{<0}$. Let $G=(V, E)$ be a graph and let $v \in V$ be one of its vertices. Let $T_{S A W}(v, G)$ be the self-avoiding walk tree of $G$ rooted at $v$. Then,

$$
p_{v}(G, \gamma)=p_{v}\left(T_{S A W}(v, G), \gamma\right)
$$

\section{FPTAS for graphs with bounded connective constant}

In this section, we prove Theorem 5. Consider $\gamma \in \mathbb{C} \backslash \mathbb{R}_{<0}$.

We will use the correlation decay technique of Weitz [16], which we adapt for use with complex activities. We review the basic idea behind the technique (see, e.g., [3, 14, 13]). For a graph $G$ (of bounded connective constant), we first express $Z_{G}(\gamma)$ as a telescoping product

$$
Z_{G}(\gamma)=1 / \prod_{i=1}^{n} p_{v_{j}}\left(G_{j}, \gamma\right)
$$

where $v_{1}, \ldots, v_{n}$ is an arbitrary enumeration of the vertices of the graph $G$ and $G_{j}$ is the graph obtained from $G$ by deleting the vertices $v_{1}, \ldots, v_{j}$. In light of (1), we can therefore focus on approximating the value $p_{v}(G, \gamma)$ for a graph $G$ and vertex $v$. Using Godsil's Theorem (cf. Theorem 9), it in turn suffices to approximate $p_{v}\left(T_{S A W}(v, G), \gamma\right)$. This might seem as a somewhat simpler task given that $T_{S A W}(v, G)$ is a tree; the caveat however is that the tree $T_{S A W}(v, G)$ is prohibitively large, so in order to be able to perform computations efficiently we need to truncate the tree. The correlation decay technique analyses the approximation error introduced by this truncation process by recursively tracking the error using tree recurrences.

In the case of matchings, for a tree $T$ and a vertex $v$ in $T$, we can write a recursion for $p_{v}(T, \gamma)$ as follows. If $v$ is the only vertex in $T$, then $p_{v}(T, \gamma)=1$ (since the only possible matching is the empty set and thus $\left.Z_{T, \neg v}(\gamma)=Z_{T}(\gamma)=1\right)$. Otherwise, let $T_{1}, \ldots, T_{d}$ be the trees of $T \backslash\{v\}$ and let $v_{1}, \ldots, v_{d}$ be the neighbours of $v$ in $T_{1}, \ldots, T_{d}$, respectively. Then, we have that

$$
Z_{T, \neg v}(\gamma)=\prod_{i=1}^{d} Z_{T_{i}}(\gamma), \quad Z_{T}(\gamma)=\prod_{i=1}^{d} Z_{T_{i}}(\gamma)+\sum_{i=1}^{d} \gamma Z_{T_{i}, \neg v_{i}}(\gamma) \prod_{j \in\{1, \ldots, d\}, j \neq i} Z_{T_{j}}(\gamma)
$$

and therefore

$$
p_{v}(T, \gamma)=\frac{Z_{T, \neg v}(\gamma)}{Z_{T}(\gamma)}=\frac{1}{1+\gamma \sum_{i=1}^{d} \frac{Z_{T_{i}, \neg v_{i}}(\gamma)}{Z_{T_{i}}(\gamma)}}=\frac{1}{1+\gamma \sum_{i=1}^{d} p_{v_{i}}\left(T_{i}, \gamma\right)}
$$


Hence, we need to evaluate the recurrence

$$
x=F\left(x_{1}, \ldots, x_{d}\right) \text { where } F\left(x_{1}, \ldots, x_{d}\right)=\frac{1}{1+\gamma \sum_{i=1}^{d} x_{i}},
$$

with base case $x=1$.

To show the decay of correlations, one wants to show that after applying the recurrence starting from two different sets of values at $v_{1}, \ldots, v_{d}$, the two computed values at $v$ will be "closer" than were the initial values at the $v_{i}$ 's. This leads us to define a notion of distance. Often straightforward distances do not suffice to show decay of correlations, and distances defined via a "potential" function are used. We adapt this notion to the complex plane.

\subsection{Metrics for measuring the error in the complex plane}

We use a distance metric based on conformal density functions (see [10] for details).

- Definition 10 (Length, Distance, Metric). Let $U$ be a simply connected open subset of $\mathbb{C}$ and let $\Phi: U \rightarrow \mathbb{R}_{>0}$ be a function (called conformal density). The length with respect to $\Phi$ of a $\operatorname{path}^{3} \eta:[0,1] \rightarrow U$ is defined as

$$
\int_{0}^{1} \Phi(\eta(t))\left|\frac{\partial}{\partial t} \eta(t)\right| d t
$$

The distance with respect to $\Phi$ between two points $x, y \in U$, denoted $\operatorname{dist}_{\Phi}(x, y)$, is the infimum of the lengths of the paths $\eta$ connecting $x$ to $y$ (that $i s, \eta(0)=x$ and $\eta(1)=y)$. We will refer to the metric induced by the distance function $\operatorname{dist}_{\Phi}(\cdot, \cdot)$ as the (conformal) metric given by $\Phi$.

We first quantify one-level correlation decay.

- Lemma 12. Let $U$ be a simply connected open subset of $\mathbb{C}, \Phi: U \rightarrow \mathbb{R}_{>0}$ be a conformal density function, and $\operatorname{dist}_{\Phi}(\cdot, \cdot)$ be the metric given by $\Phi$. Let $p$ and $q$ be conjugate exponents, that is, $1 / p+1 / q=1$, where $p, q \in \mathbb{R}_{>0} \cup\{\infty\}$.

Suppose that $d \geq 1$ is an integer and $F: U^{d} \rightarrow U$ is a holomorphic map. Let $x_{1}, \ldots, x_{d} \in$ $U$ and $y_{1}, \ldots, y_{d} \in U$ and let $x=F\left(x_{1}, \ldots, x_{d}\right)$ and $y=F\left(y_{1}, \ldots, y_{d}\right)$. Assume that there exists a real $\alpha \in(0,1)$ such that for any $z_{1}, \ldots, z_{d} \in U$

$$
\sum_{i=1}^{d}\left|\Phi\left(F\left(z_{1}, \ldots, z_{d}\right)\right) \frac{\partial F}{\partial z_{i}}\left(z_{1}, \ldots, z_{d}\right) \frac{1}{\Phi\left(z_{i}\right)}\right|^{p} \leq \alpha^{p} .
$$

Then

$$
\operatorname{dist}_{\Phi}(x, y) \leq \alpha\left(\sum_{i=1}^{d} \operatorname{dist}_{\Phi}\left(x_{i}, y_{i}\right)^{q}\right)^{1 / q}
$$

Proof. Let $\epsilon>0$. For $i \in[d]$, let $\eta_{i}$ be a path connecting $x_{i}$ to $y_{i}$ of length $\ell_{i} \leq \operatorname{dist}_{\Phi}\left(x_{i}, y_{i}\right)+\epsilon$. We assume w.l.o.g. that $\eta_{i}$ is re-parameterized to uniform speed (using arc length), that is, for a.e. $t \in[0,1]$ we have

$$
\left|\frac{\partial}{\partial t} \eta_{i}(t)\right| \Phi\left(\eta_{i}(t)\right)=\ell_{i}
$$

${ }^{3}$ Following [10], paths are assumed to be continuous and piecewise continuously differentiable. 
We now define a path $\eta$ connecting $x$ to $y$ :

$$
\eta(t):=F\left(\eta_{1}(t), \ldots, \eta_{d}(t)\right) .
$$

Let $L$ denote the length of $\eta$ and $F_{i}\left(x_{1}, \ldots, x_{d}\right)$ denote the function $\frac{\partial F}{\partial x_{i}}\left(x_{1}, \ldots, x_{d}\right)$ Then, using the triangle inequality and (5), we have

$$
\begin{aligned}
L & =\int_{0}^{1} \Phi(\eta(t))\left|\frac{\partial}{\partial t} \eta(t)\right| d t=\int_{0}^{1} \Phi(\eta(t))\left|\sum_{i=1}^{d} F_{i}\left(\eta_{1}(t), \ldots, \eta_{d}(t)\right) \frac{\partial \eta_{i}}{\partial t}(t)\right| d t \\
& \leq \int_{0}^{1} \Phi(\eta(t)) \sum_{i=1}^{d}\left|F_{i}\left(\eta_{1}(t), \ldots, \eta_{d}(t)\right) \frac{\partial \eta_{i}}{\partial t}(t)\right| d t \\
& =\int_{0}^{1} \sum_{i=1}^{d}\left|\Phi(\eta(t)) F_{i}\left(\eta_{1}(t), \ldots, \eta_{d}(t)\right) \frac{1}{\Phi\left(\eta_{i}(t)\right)} \ell_{i}\right| d t .
\end{aligned}
$$

By Hölder's inequality and condition (3), for any $t \in[0,1]$, we have

$$
\begin{aligned}
& \sum_{i=1}^{d}\left|\Phi(\eta(t)) F_{i}\left(\eta_{1}(t), \ldots, \eta_{d}(t)\right) \frac{1}{\Phi\left(\eta_{i}(t)\right)} \ell_{i}\right| \leq \\
& \quad\left(\sum_{i=1}^{d}\left|\Phi(\eta(t)) F_{i}\left(\eta_{1}(t), \ldots, \eta_{d}(t)\right) \frac{1}{\Phi\left(\eta_{i}(t)\right)}\right|^{p}\right)^{1 / p}\left(\sum_{i=1}^{d} \ell_{i}^{q}\right)^{1 / q} \leq \alpha\left(\sum_{i=1}^{d} \ell_{i}^{q}\right)^{1 / q} .
\end{aligned}
$$

Integrating this for $t$ between 0 and 1 and combining with (6), we obtain

$$
\operatorname{dist}_{\Phi}(x, y) \leq L \leq \alpha\left(\sum_{i=1}^{d} \ell_{i}^{q}\right)^{1 / q} .
$$

Taking $\epsilon \rightarrow 0$ we obtain

$$
\operatorname{dist}_{\Phi}(x, y) \leq \alpha\left(\sum_{i=1}^{d} \operatorname{dist}_{\Phi}\left(x_{i}, y_{i}\right)^{q}\right)^{1 / q} .
$$

Now, given a rooted tree, our goal will be to bound the correlation decay at the root when we truncate the tree at $\operatorname{depth} \Theta(\log n)$. Let $T$ be a finite tree rooted at a vertex $\rho$ and let $C$ be a subset of the leaves of $T$. Let $U \subseteq \mathbb{C}$. We will have a family of maps $\left\{F_{d}\right\}_{d \geq 1}$ where $F_{d}: U^{d} \mapsto U$ will be a symmetric map of arity $d$ (which will be the recurrence applied to a vertex of the tree with $d$ children). Let $\sigma: C \rightarrow U$ be an arbitrary assignment of values in $U$ to the vertices of $C$. Let also $u_{0} \in U$ be the "initial" value ( $u_{0}$ corresponds to the starting point of the recurrences). For a vertex $v$ in $T$ and an initial value $u_{0} \in U$, we define the quantity $r_{v}\left(C, \sigma, u_{0}\right)$ recursively as follows.

$$
r_{v}\left(C, \sigma, u_{0}\right)= \begin{cases}u_{0} & \text { if } v \text { is a leaf of } T \text { and } v \notin C, \\ \sigma(v) & \text { if } v \in C, \\ F_{d}\left(x_{1}, \ldots, x_{d}\right) & \text { otherwise, where } x_{i}=r_{v_{i}}\left(C, \sigma, u_{0}\right) \\ & \text { and } v_{1}, \ldots, v_{d} \text { are } v \text { 's children in } T .\end{cases}
$$

We can now study the sensitivity of $r_{v}\left(C, \sigma, u_{0}\right)$ to the assignment $\sigma$. The following lemma is the analogue of [14, Lemma 3] for the complex plane and will be used to apply the correlation decay technique for graphs of bounded connective constant, the proof can be found in the full version. 
- Lemma 13. Let $U$ be a simply connected open subset of $\mathbb{C}$ and $\Phi: U \rightarrow \mathbb{R}_{>0}$ be a conformal density function. For $d=1,2, \ldots$, let $F_{d}: U^{d} \mapsto U$ be symmetric holomorphic maps. Suppose that there exists a real $\alpha \in(0,1)$ and conjugate exponents $p$ and $q$ such that for every integer $d \geq 1$ and all $z_{1}, \ldots, z_{d} \in U$ it holds that

$$
\sum_{i=1}^{d}\left|\Phi\left(F_{d}\left(z_{1}, \ldots, z_{d}\right)\right) \frac{\partial F_{d}}{\partial z_{i}}\left(z_{1}, \ldots, z_{d}\right) \frac{1}{\Phi\left(z_{i}\right)}\right|^{p} \leq \alpha^{p} .
$$

Then, the following holds for any initial value $u_{0} \in U$ and any finite tree $T$ rooted at $\rho$.

Let $C$ be a subset of the leaves of $T$ and consider two arbitrary assignments $\sigma_{1}: C \rightarrow U$ and $\sigma_{2}: C \rightarrow U$. Then

$$
\left|r_{\rho}\left(C, \sigma_{1}, u_{0}\right)-r_{\rho}\left(C, \sigma_{2}, u_{0}\right)\right| \leq\left(\frac{M}{L}\right)\left(\sum_{v \in C} \alpha^{q \cdot \operatorname{depth}(v)}\right)^{1 / q},
$$

where $L:=\inf _{x \in U} \Phi(x), M:=\max _{v \in C} \operatorname{dist}_{\Phi}\left(\sigma_{1}(v), \sigma_{2}(v)\right)$ and $\operatorname{depth}(v)$ is the distance of $v$ from the root $\rho$.

\subsection{Applying the method for matchings}

Suppose that $\gamma \in \mathbb{C} \backslash \mathbb{R}_{\leq 0}$. We will parameterise $\gamma$ as

$$
\gamma=(1 / Q)^{2}, \text { where we choose } Q \text { such that } \operatorname{Re}(Q)>0 .
$$

Note that, in the choice of $Q$, we used the assumption that $\gamma$ is not a negative real number. Let $\mathcal{H}$ be the right complex half-plane, that is, the set of complex $x$ such that $\operatorname{Re}(x)>0$, and note that $Q \in \mathcal{H}$. We will also transform the space in which the quantities $p_{v}(G, \gamma)$ live using the map $x \mapsto x / Q$. In the transformed space, the recurrence (2) becomes

$$
y=F\left(y_{1}, \ldots, y_{d}\right) \text { where } F\left(y_{1}, \ldots, y_{d}\right)=\frac{1}{Q+\sum_{i=1}^{d} y_{i}},
$$

where if $y$ corresponds to a leaf then $y=1 / Q$ (we refer to this $y$ as the initial $y$ ). Let

$$
U=\{y \in \mathbb{C}|\operatorname{Re}(y)>0,| y \mid<1 / \operatorname{Re}(Q)\} .
$$

The following lemma shows that the set $U$ is closed under application of the recurrence (10).

- Lemma 14. Suppose that $y_{1}, \ldots, y_{d} \in U$ and $\operatorname{Re}(Q)>0$. Then, for $y$ given by (10), we have that $y \in U$ as well. In fact, we have that $\operatorname{Re}(y) \geq \frac{\operatorname{Re}(Q)}{\left(|Q|+\frac{d}{\operatorname{Re}(Q)}\right)^{2}}$.

We next go on to show the required contraction properties for an appropriate function $\Phi$. This is largely based on arguments from [13] from the real case, which we can adapt to the complex plane to obtain the following.

- Lemma 16. Let $\Delta$ be a positive real number, $\gamma \in \mathbb{C} \backslash \mathbb{R}_{<0}$, and $Q, U$ be given from (9) and (11), respectively. Consider the function $\Phi: U \mapsto \mathbb{R}_{>0}$ given by $\Phi(y)=\frac{1}{\operatorname{Re}(y)(2 / \operatorname{Re}(Q)-\operatorname{Re}(y))}$ and let $\hat{\gamma}=\frac{2|\gamma|}{1+\cos (\arg \gamma)}$,

$$
D=\max \left\{\Delta, \frac{3}{4 \hat{\gamma}}\right\}, \quad p=1 /\left(1-\frac{1}{\sqrt{1+4 \hat{\gamma} D}}\right), \quad q=\frac{p}{p-1}, \quad \alpha=\frac{1}{D^{1 / q}}\left(1-\frac{2}{1+\sqrt{1+4 \hat{\gamma} D}}\right) .
$$


Then, the following holds for all integer $d \geq 1$.

Consider the map $F: U^{d} \mapsto U$ given by $F\left(y_{1}, \ldots, y_{d}\right)=\frac{1}{Q+\sum_{i=1}^{d} y_{i}}$. Then, for arbitrary $y_{1}, \ldots, y_{d} \in U$ we have

$\sum_{i=1}^{d}\left|\Phi\left(F\left(y_{1}, \ldots, y_{d}\right)\right) \frac{\partial F}{\partial y_{i}}\left(y_{1}, \ldots, y_{d}\right) \frac{1}{\Phi\left(y_{i}\right)}\right|^{p} \leq \alpha^{p}$

Based on the above lemmas, we can now give a proof sketch of Theorem 5 .

Proof Sketch of Theorem 5. If $\gamma$ is a non-negative real number, then the result follows from [13, Theorem 1.3]. So we focus on the case where $\gamma$ is not real. Using the telescoping expansion of $Z_{G}(\gamma)$ described in (1), it suffices to give an algorithm that on an input graph $G \in \mathcal{F}_{\Delta, a, c}$, a vertex $v$ in $G$ and rational $\delta>0$ outputs in time $(\hat{c} n / \delta)^{O((1+a+\sqrt{|\hat{\gamma}| \Delta}) \log \Delta)}$ a quantity $\tilde{p}$ which satisfies $\tilde{p}=p_{v}(G, \gamma) \mathrm{e}^{z}$ for some complex number $z$ with $|z| \leq \delta$.

Let $T=T_{S A W}(v, G)$ be the self-avoiding walk tree rooted at $v$, then by Theorem 9 we have that $p_{v}(G, \gamma)=p_{v}(T, \gamma)$, so it suffices to approximate $p_{v}(T, \gamma)$. For this, we apply the general framework of Lemma 13 to the recurrence in (10) using the contraction properties proved in Lemma 16. More precisely, we first truncate the tree $T$ at logarithmic depth $\ell$ to obtain a tree $T^{\prime}$ and we output $\hat{p}=p_{v}\left(T^{\prime}, \gamma\right)$ as our approximation to $p_{v}(T, \gamma)$. Note that $T^{\prime}$ has size at most $c \Delta^{\ell}$ since $G$ has connective constant at most $\Delta$. We then invoke Lemmas 13 and 16 to show that the absolute error between $p_{v}(T, \gamma)$ and $p_{v}\left(T^{\prime}, \gamma\right)$ decays as $\left(\Delta^{1 / q} \alpha\right)^{\ell}$ where $\alpha<1 / \Delta^{1 / q}$ is the constant in Lemma 16. By taking $\ell=\Theta(\log n)$, we can therefore make the absolute error as small as an inverse polynomial in $n$. The absolute error can then be translated to the desired relative error between $\hat{p}$ and $p_{v}(G, \gamma)$ using the bound in Lemma 14.

\section{Proof of hardness results}

Let $\gamma_{0}=-1 / 10$ and $\mathcal{G}$ be the set of graphs of maximum degree 3 . It is well-known [5, Theorem 3] that the problem of exactly computing $Z_{G}\left(\gamma_{0}\right)$ given an input graph $G \in \mathcal{G}$ is \#P-hard. Moreover, by Corollary 8 we have that $Z_{G}\left(\gamma_{0}\right)>0$ for all graphs $G \in \mathcal{G}$.

Using an oracle on graphs $H$ of maximum degree $\Delta$ for either approximating $Z_{H}(\gamma)$ multiplicatively or deciding the sign of $Z_{H}(\gamma)$, we will design a polynomial time algorithm to exactly compute the ratio $\frac{Z_{G}\left(\gamma_{0}\right)}{Z_{G-e^{*}\left(\gamma_{0}\right)}}$ for an arbitrary graph $G \in \mathcal{G}$ and an arbitrary edge $e^{*}$ of $G$; note that this ratio is well-defined since $Z_{G-e^{*}}\left(\gamma_{0}\right)>0$. With such a subroutine at hand, we can compute $Z_{G}\left(\gamma_{0}\right)$ using self-reducibility techniques; namely, let $e_{1}, e_{2}, \ldots, e_{m}$ be an enumeration of the edges of $G$ and let $G_{i}$ be the graph where the edges $e_{i}, \ldots, e_{m}$ are deleted (note that $G_{m+1}=G$ and $G_{1}$ is the empty graph). Then, we have that $Z_{G}\left(\gamma_{0}\right)=\prod_{i=1}^{m} \frac{Z_{G_{i+1}}\left(\gamma_{0}\right)}{Z_{G_{i}}\left(\gamma_{0}\right)}$. This yields the \#P-hardness results of Theorems 1 and 2 .

The most difficult part of designing the subroutine is constructing graph gadgets that have the effect of "changing" the edge activity $\gamma$ to any desired activity, perhaps with some small error. It is actually important to make the error exponentially small relative to the size of the graph. To formalise these gadget constructions, we will need some definitions. Let $G=(V, E)$ be a graph and $u, v \in V$. Analogously to the notation $Z_{G, u}(\gamma)$ and $Z_{G, \neg u}(\gamma)$ of Section 2, we let $Z_{G, u, v}(\gamma)$ be the contribution to the partition function $Z_{G}(\gamma)$ from those matchings $M \in \mathcal{M}_{G}$ such that both $u, v$ are matched in $M$, and we define $Z_{G, u, \neg v}(\gamma), Z_{G, \neg u, v}(\gamma), Z_{G, \neg u, \neg v}(\gamma)$ similarly. 
- Definition 17. Fix a real number $\gamma$. Given $\gamma$, the graph $G=(V, E)$ is said to implement the edge activity $\gamma^{\prime} \in \mathbb{R}$ with accuracy $\epsilon>0$ if there are vertices $u, v$ in $G$ such that $Z_{G, \neg u, \neg v}(\gamma) \neq 0$ and

1. $u, v$ have degree one in $G$ and $(u, v) \notin E$,

2. $\left|\frac{Z_{G, u, \neg v}(\gamma)}{Z_{G, \neg u, \neg v}(\gamma)}\right| \leq \epsilon,\left|\frac{Z_{G, \neg u, v}(\gamma)}{Z_{G, \neg u, \neg v}(\gamma)}\right| \leq \epsilon$,

3. $\left|\frac{Z_{G, u, v}(\gamma)}{Z_{G, \neg u, \neg v}(\gamma)}-\gamma^{\prime}\right| \leq \epsilon$.

We call $u, v$ the terminals of $G$. If both of Items 2 and 3 hold with $\epsilon=0$, we say that $G$ implements the edge activity $\gamma^{\prime}$ (perfectly).

- Definition 18. Let $\alpha$ be a rational number and write $\alpha=p / q$, where $p, q$ are integers such that $\operatorname{gcd}(p, q)=1$. Then, the size of $\alpha$, denoted by $\operatorname{size}(\alpha)$, is given by $1+\log (|p|+|q|)$. For $\alpha_{1}, \ldots, \alpha_{t} \in \mathbb{Q}$, we denote by $\operatorname{size}\left(\alpha_{1}, \ldots, \alpha_{t}\right)$ the total of the sizes of $\alpha_{1}, \ldots, \alpha_{t}$.

Our key lemma for designing the subroutine is the following.

- Lemma 19. Let $\Delta \geq 3$ be an integer and $\gamma<-\frac{1}{4(\Delta-1)}$ be a rational number.

There is an algorithm which, on input rational $\gamma^{\prime} \leq 0$ and $\epsilon>0$, outputs in poly $\left(\operatorname{size}\left(\gamma^{\prime}, \epsilon\right)\right)$ time a bipartite graph $G$ of maximum degree at most $\Delta$ with terminals $u, v$ in the same part of the vertex partition of $G$ so that $G$ implements $\gamma^{\prime}$ with accuracy $\epsilon$.

In turn, to prove Lemma 19 it will be simpler to first construct graph gadgets that implement "vertex" activities.

- Definition 22. Fix a real number $\gamma$. Given $\gamma$, the graph $G=(V, E)$ is said to implement the vertex activity $\lambda \in \mathbb{R}$ with accuracy $\epsilon>0$ if there is vertex $u$ in $G$ such that

1. $u$ has degree one in $G$,

2. $Z_{G}(\gamma) \neq 0$ and $\left|\frac{Z_{G, \neg u}(\gamma)}{Z_{G}(\gamma)}-\lambda\right| \leq \epsilon$.

We call $u$ the terminal of $G$. If Item 2 holds with $\epsilon=0$, we say that $G$ implements $\lambda$ (perfectly).

Our main lemma about implementing vertex activities is as follows.

- Lemma 23. Let $\Delta \geq 3$ be an integer and $\gamma<-\frac{1}{4(\Delta-1)}$ be a rational number.

There is an algorithm which, on input a rational number $\lambda$ and $\epsilon>0$, outputs in poly $(\operatorname{size}(\lambda, \epsilon))$ time a bipartite graph $G$ of maximum degree at most $\Delta$ that implements the vertex activity $\lambda$ with accuracy $\epsilon$.

In order to obtain the exponential precision of Lemma 23, we will first show how to implement vertex activities with arbitrarily small constant precision, as formalised in the following lemma.

- Lemma 24. Let $\Delta \geq 3$ be an integer and $\gamma<-\frac{1}{4(\Delta-1)}$ be a real number.

For every $\lambda \in \mathbb{R}$ and $\epsilon>0$, there is a bipartite graph $G$ of maximum degree at most $\Delta$ that implements the vertex activity $\lambda$ with accuracy $\epsilon$.

To prove Lemma 24, we will need to consider two cases for the value of $\gamma$. Namely, for an integer $\Delta \geq 3$, the following subset of the negative reals will be relevant:

$\mathcal{B}_{\Delta}=\left\{\gamma \in \mathbb{R} \mid \gamma=-\frac{1}{4(\Delta-1)(\cos \theta)^{2}}\right.$ for some $\theta \in(0, \pi / 2)$ that is a rational multiple of $\left.\pi\right\}$. 
If $\gamma \notin \mathcal{B}_{\Delta}$ then we show that we can use $(\Delta-1)$-ary trees of appropriate height to obtain the constant accuracy of Lemma 24. The situation is more complicated for $\gamma \in \mathcal{B}_{\Delta}$ since the $(\Delta-1)$-ary tree is not as effective; neverthless, it can still be used to show that we can implement perfectly the edge activity $\gamma^{\prime}=-1$ (though in some cases we have to work a bit harder, cf. Lemmas 25 and 27 of the full version). To make use of $\gamma^{\prime}=-1$, we show in Lemma 26 of the full version that for every rational number $\lambda$, there exists a tree of maximum degree $\Delta=3$ that implements the vertex activity $\lambda$ and this can then be propagated to get the constant accuracy of Lemma 24 for $\gamma \in \mathcal{B}_{\Delta}$.

We then bootstrap Lemma 24 to obtain the exponential precision required in Lemma 23, based on the "contracting maps that cover" technique of [4]. The key is to build a finite collection of maps $\Phi_{i}: x \mapsto \frac{1}{1+\gamma\left(\lambda_{i}+x\right)}$ for different values of $\lambda_{i}$ (obtained from Lemma 24) and to apply these iteratively to amplify precision on an appropriately chosen interval of the real axis; the details of the construction as well as the choice of the $\lambda_{i}$ 's (see Lemma 30 below) depend heavily on the fact that we are working with the matching polynomial. Using the maps $\Phi_{i}$, Lemma 23 can then be proved using a careful analysis depending on the value of $\lambda$ (relative to the interval) and, once this is in place, we have everything we need to prove Lemma 19, see the full version for details.

The proof of the hardness results for graphs with bounded connective constant (Theorem 4) can be obtained by adapting our arguments above. Namely, we let $S=\bigcup_{d \geq 3} \mathcal{B}_{d}$. Then, for $\gamma \in S$, as we discussed earlier, there exists a tree that implements the edge activity $\gamma^{\prime}=-1$. We can then modify the tree so that the terminals of the final tree are at distance $\ell$, for arbitrarily large $\ell$. The key now is that we can attach the new tree to the edges of a target graph to modify the edge activity and at the same time reduce its connective constant (since there is just one path connecting the terminals of the tree gadget). Since $S$ is dense on the negative real axis, we therefore obtain Theorem 4 by applying the hardness results of Theorems 1 and 2.

We conclude by giving the deferred construction of the maps $\Phi_{i}$ which are used to obtain the exponential precision of Lemma 23. The proof of the following lemma establishes important properties of the maps that enable them to bootstrap precision. The lemma also gives an algorithm that can be used to implement any "target" $y$ with exponential precision. ${ }^{4}$

- Lemma 30. Let $\gamma<0$ be a rational number. Then, there exist rationals $x_{0}$ and $r, \delta>0$ and reals $\lambda_{1}^{*}, \ldots, \lambda_{t}^{*}$ (for some positive integer $t$ ) such that the following holds for all rational $\lambda_{1}, \ldots, \lambda_{t}$ satisfying $\left|\lambda_{i}-\lambda_{i}^{*}\right| \leq \delta$ for $i \in[t]$.

Let $I:=\left[x_{0}-r, x_{0}+r\right]$ and, for $i \in[t]$, consider the map $\Phi_{i}: x \mapsto \frac{1}{1+\gamma\left(\lambda_{i}+x\right)}$ for $x \neq-\left(1+\gamma \lambda_{i}\right) / \gamma$. There is an algorithm which, on input (i) a starting point $y_{0} \in I \cap \mathbb{Q}$, (ii) a target $y \in I \cap \mathbb{Q}$, and (iii) a rational $\epsilon>0$, outputs in poly $\left(\operatorname{size}\left(y_{0}, y, \epsilon\right)\right)$ time a number $\hat{y} \in I \cap \mathbb{Q}$ and a sequence $i_{1}, i_{2}, \ldots, i_{k} \in[t]$ such that

$$
\hat{y}=\Phi_{i_{k}}\left(\Phi_{i_{k-1}}\left(\cdots \Phi_{i_{1}}\left(y_{0}\right) \cdots\right)\right) \text { and }|\hat{y}-y| \leq \epsilon .
$$

Proof. Let $x_{1}, x_{2}$ be rationals such that $\gamma x_{1} x_{2}=-1$ and $x_{1} \neq \pm x_{2}$. Let $\lambda$ be such that $1+\gamma \lambda=-\gamma\left(x_{1}+x_{2}\right)$. Then, the fixpoints of the map $\Phi: x \mapsto \frac{1}{1+\gamma(\lambda+x)}$ are $x_{1}$ and $x_{2}$, and at least one of the two points is attracting. ${ }^{5}$ Denote by $x_{0}$ the attracting fixpoint of $\Phi$, so

\footnotetext{
4 The "target" $y$ corresponds to a vertex activity $\lambda$ - the only difference is that a transformation between them has been applied for technical reasons, see proof of Lemma 23 in the full version for details.

5 To see this, note that $\Phi(x)=x$ is equivalent to $x(1+\gamma \lambda)+\gamma x^{2}=1$ and therefore $x_{1}$ and $x_{2}$ are (the only) fixpoints of $\Phi$. Moreover, we have that $\Phi^{\prime}(x)=-\frac{\gamma}{(1+\gamma(\lambda+x))^{2}}$ and hence $\Phi^{\prime}\left(x_{1}\right)=-\gamma x_{1}^{2}$,
} 
that $x_{0}$ satisfies $\Phi\left(x_{0}\right)=x_{0}$ and $0<\left|\Phi^{\prime}\left(x_{0}\right)\right|<1$. By Lemma 21 of the full version, we can compute $\eta>0$ such that for all $x \in\left[x_{0}-\eta, x_{0}+\eta\right]$ and all $\lambda^{\prime} \in[\lambda-\eta, \lambda+\eta]$ it holds that

$$
1+\gamma\left(\lambda^{\prime}+x\right) \neq 0 \text { and }\left|\frac{\gamma}{\left(1+\gamma\left(\lambda^{\prime}+x\right)\right)^{2}}-\frac{\gamma}{\left(1+\gamma\left(\lambda+x_{0}\right)\right)^{2}}\right| \leq \frac{1}{2} \min \left\{\left|\Phi^{\prime}\left(x_{0}\right)\right|, 1-\left|\Phi^{\prime}\left(x_{0}\right)\right|\right\} .
$$

Let $r:=\frac{\left|\Phi^{\prime}\left(x_{0}\right)\right|}{4} \eta, \delta:=(r / 4)$ and let $\lambda_{1}^{*}, \ldots, \lambda_{t}^{*}$ form a $\delta$-covering of the interval $[\lambda-\eta / 2, \lambda+$ $\eta / 2]$. Let $\lambda_{1}, \ldots, \lambda_{t}$ be arbitrary rationals satisfying $\left|\lambda_{i}-\lambda_{i}^{*}\right| \leq \delta$. For $i \in[t]$ consider the maps $\Phi_{i}: x \mapsto \frac{1}{1+\gamma\left(\lambda_{i}+x\right)}$. Finally, let $I$ be the interval $\left[x_{0}-r, x_{0}+r\right]$. We will show

Property 1: The maps $\left\{\Phi_{i}\right\}_{i \in[t]}$ are contracting on the interval $I$, and

Property 2: $I \subseteq \Phi_{1}(I) \cup \cdots \cup \Phi_{t}(I)$.

Once these two properties of the maps $\left\{\Phi_{i}\right\}_{i \in[t]}$ are proved, the algorithm in the statement of the lemma and its analysis are almost identical to those in [4, Proof of Lemmas 12 \& 26]. The only difference here is that the maps $\left\{\Phi_{i}\right\}_{i \in[t]}$ have different expressions. The fact that we need about the expression of the maps is that, for $i \in[t]$ and for every rational $x, \Phi_{i}^{-1}(x)$ can be computed in time poly $\left(\operatorname{size}\left(x, \lambda_{i}, \gamma\right)\right)$. This is clear since $\Phi_{i}^{-1}(x)=\frac{1}{\gamma}\left(\frac{1}{x}-1\right)-\lambda_{i}$.

Proof of Property 1. Fix $i \in[t]$. We will show that $\Phi_{i}$ is contracting on the interval $I$. Observe that $r<\eta / 4$ since $\left|\Phi^{\prime}\left(x_{0}\right)\right|<1$ and therefore $\delta<\eta / 4$ as well. Then, we have by the triangle inequality that

$$
\left|\lambda_{i}-\lambda\right| \leq\left|\lambda_{i}-\lambda_{i}^{*}\right|+\left|\lambda_{i}^{*}-\lambda\right| \leq \delta+\eta / 2<\eta .
$$

Therefore, we can apply (15) to $\lambda^{\prime}=\lambda_{i}$ and $x \in I$. Observe that $\Phi^{\prime}(x)=-\gamma /\left(1+\gamma\left(\lambda_{i}+x\right)\right)^{2}$ and $\Phi^{\prime}\left(x_{0}\right)=-\gamma /\left(1+\gamma\left(\lambda_{i}+x_{0}\right)\right)^{2}$ and hence we obtain that for all $x \in I$ it holds that

$$
\left|\Phi_{i}^{\prime}(x)\right| \leq \frac{1}{2}\left(1+\left|\Phi^{\prime}\left(x_{0}\right)\right|\right)<1 .
$$

It follows that the maps $\Phi_{i}$ are contracting on the interval $I$ for all $i \in[t]$.

Proof of Property 2. It suffices to consider an arbitrary $y \in I$ and show that there exists $j \in[t]$ such that $\Phi_{j}^{-1}(y) \in I$. To do this, we set $J$ to be the interval $\left[x_{0}-\eta / 2, x_{0}+\eta / 2\right]$ and consider the map $\Phi$ on the interval $J$. Then, (15) for $\lambda^{\prime}=\lambda$ and $x \in J$ gives that

$$
0<\frac{1}{2}\left|\Phi^{\prime}\left(x_{0}\right)\right| \leq\left|\Phi^{\prime}(x)\right|
$$

and therefore, by the Mean Value Theorem, for $z, w \in J$ we have that

$$
\frac{1}{2}\left|\Phi^{\prime}\left(x_{0}\right)\right| \cdot|z-w| \leq|\Phi(z)-\Phi(w)| .
$$

We thus have that

$$
\begin{aligned}
& \left|\Phi\left(x_{0}+\eta / 2\right)-x_{0}\right|=\left|\Phi\left(x_{0}+\eta / 2\right)-\Phi\left(x_{0}\right)\right| \geq \eta\left|\Phi^{\prime}\left(x_{0}\right)\right| / 4=r \\
& \left|\Phi\left(x_{0}-\eta / 2\right)-x_{0}\right|=\left|\Phi\left(x_{0}-\eta / 2\right)-\Phi\left(x_{0}\right)\right| \geq \eta\left|\Phi^{\prime}\left(x_{0}\right)\right| / 4=r .
\end{aligned}
$$

Since $\Phi$ is monotonically increasing and continuous on the interval $J$, we therefore obtain that $I \subseteq \Phi(J)$. Therefore, for arbitrary $y \in I$ it holds that $\Phi^{-1}(y) \in J$ and hence from (16) applied to $z=\Phi^{-1}(y)$ and $w=\Phi^{-1}\left(x_{0}\right)$, we obtain that

$$
\left|\Phi^{-1}(y)-x_{0}\right|=\left|\Phi^{-1}(y)-\Phi^{-1}\left(x_{0}\right)\right| \leq\left(2 /\left|\Phi^{\prime}\left(x_{0}\right)\right|\right)\left(y-x_{0}\right) \leq \eta / 2 .
$$

$\Phi^{\prime}\left(x_{2}\right)=-\gamma x_{2}^{2}$. Therefore $\left|\Phi^{\prime}\left(x_{1}\right)\right| \neq\left|\Phi^{\prime}\left(x_{2}\right)\right|$ and $1=\left|\gamma x_{1} x_{2}\right|=\sqrt{\left|\Phi^{\prime}\left(x_{1}\right)\right|\left|\Phi^{\prime}\left(x_{2}\right)\right|}$. Therefore either $\left|\Phi^{\prime}\left(x_{1}\right)\right|<1$ or $\left|\Phi^{\prime}\left(x_{2}\right)\right|<1$. 
Since $\lambda_{1}^{*}, \ldots, \lambda_{t}^{*}$ is a $\delta$-covering of the interval $[\lambda-\eta / 2, \lambda+\eta / 2]$, it follows that there exists $j \in[t]$ such that

$$
\left|\lambda+\Phi^{-1}(y)-x_{0}-\lambda_{j}^{*}\right| \leq \delta=r / 4 .
$$

Now, observe that $\Phi_{j}^{-1}(y)=\frac{1}{\gamma}\left(\frac{1}{y}-1\right)-\lambda_{j}$ and $\Phi^{-1}(y)=\frac{1}{\gamma}\left(\frac{1}{y}-1\right)-\lambda$, so we have that

$$
\begin{aligned}
\left|\Phi_{j}^{-1}(y)-x_{0}\right| & =\left|\frac{1}{\gamma}\left(\frac{1}{y}-1\right)-\lambda_{j}-x_{0}\right|=\left|\lambda+\Phi^{-1}(y)-x_{0}-\lambda_{j}\right| \\
& \leq\left|\lambda+\Phi^{-1}(y)-x_{0}-\lambda_{j}^{*}\right|+\left|\lambda_{j}-\lambda_{j}^{*}\right| \leq r / 4+r / 4=r / 2 .
\end{aligned}
$$

It follows that $y \in \Phi_{j}(I)$ and therefore, since $y$ was arbitrary, we have that $I \subseteq \Phi_{1}(I) \cup \cdots \cup$ $\Phi_{t}(I)$.

This completes the proof of Properties 1 and 2, and hence the proof of Lemma 30.

\section{References}

1 A. Barvinok. Computing the Permanent of (Some) Complex Matrices. Foundations of Computational Mathematics, 16(2):329-342, 2016.

2 A. Barvinok. Combinatorics and Complexity of Partition Functions. Algorithms and Combinatorics. Springer International Publishing, 2017.

3 M. Bayati, D. Gamarnik, D. A. Katz, C. Nair, and P. Tetali. Simple Deterministic Approximation Algorithms for Counting Matchings. In Proceedings of the 39th Annual ACM Symposium on Theory of Computing, San Diego, California, USA, June 11-13, 2007, pages 122-127, 2007.

4 I. Bezáková, A. Galanis, L. A. Goldberg, and D. Štefankovič. Inapproximability of the Independent Set Polynomial in the Complex Plane. In Proceedings of the 50th Annual ACM SIGACT Symposium on Theory of Computing, STOC 2018, pages 1234-1240, 2018. Full version available from arxiv:1711.00282.

5 J.-Y. Cai, S. Huang, and P. Lu. From Holant to \#CSP and Back: Dichotomy for Holant ${ }^{c}$ Problems. Algorithmica, 64(3):511-533, 2012.

6 C. D. Godsil. Matchings and Walks in Graphs. Journal of Graph Theory, 5(3):285-297, 1981

7 Nicholas J. A. Harvey, Piyush Srivastava, and Jan Vondrák. Computing the Independence Polynomial: from the Tree Threshold down to the Roots. In Proceedings of the Twenty-Ninth Annual ACM-SIAM Symposium on Discrete Algorithms, SODA 2018, pages 1557-1576, 2018.

8 O. J. Heilmann and E. H. Lieb. Theory of Monomer-Dimer Systems. Communications in Mathematical Physics, 25(3):190-232, 1972.

9 M. Jerrum and A. Sinclair. Approximating the Permanent. SIAM J. Comput., 18(6):1149-1178, 1989.

10 D. Kraus and O. Roth. Conformal Metrics. CoRR, abs/0805.2235, 2008. arXiv:0805.2235.

11 V. Patel and G. Regts. Deterministic Polynomial-Time Approximation Algorithms for Partition Functions and Graph Polynomials. SIAM Journal on Computing, 46(6):1893-1919, 2017.

12 H. Peters and G. Regts. On a Conjecture of Sokal Concerning Roots of the Independence Polynomial. The Michigan Mathematical Journal, 2019. To appear. URL: https: //projecteuclid.org/euclid.mmj/1541667626\#info.

13 A. Sinclair, P. Srivastava, D. Štefankovič, and Y. Yin. Spatial Mixing and the Connective Constant: Optimal Bounds. Probability Theory and Related Fields, 168(1):153-197, 2017.

14 A. Sinclair, P. Srivastava, and Y. Yin. Spatial Mixing and Approximation Algorithms for Graphs with Bounded Connective Constant. In Proceedings of the 2013 IEEE 54th Annual Symposium on Foundations of Computer Science, FOCS '13, pages 300-309, 2013.

15 B.-B. Wei, S.-W. Chen, H.-C. Po, and R.-B. Liu. Phase Transitions in the Complex Plane of Physical Parameters. Nature Scientific Reports, 4:5202; DOI: 10.1038/srep05202, 2014.

16 D. Weitz. Counting Independent Sets Up to the Tree Threshold. In Proceedings of the Thirty-eighth Annual ACM Symposium on Theory of Computing, STOC '06, pages 140-149, New York, NY, USA, 2006. ACM. 